\title{
Aplicação de técnicas compensatórias no controle dos escoamentos superficiais: estudo de caso em loteamento residencial em Jacarepaguá, Rio de Janeiro
}

\author{
Dayana Martins Nunes "; Marlon Giovanni Lopes Alvarez '; Alfredo Akira Ohnuma Jr'; Luciene \\ Pimentel da Silva ${ }^{l}$ \\ dayana.mnunes@gmail.com
}

1. Universidade do Estado do Rio de Janeiro, Rua São Francisco Xavier, 524, Rio de Janeiro, Brasil.

Histórico do Artigo:

Recebido: 16 de março de 2016

Aceito: 21 de novembro de 2017

Publicado: 18 de julho de 2017

\begin{abstract}
Resumo: 0 processo de urbanização das cidades brasileiras resultou em um adensamento das áreas urbanas e, consequentemente, aumento das áreas impermeáveis, que favorecem a ocorrência de enchentes. Para compensar os impactos da urbanização no ciclo hidrológico, são propostas medidas de controle localizadas e de pequena escala que promovem aumento da infiltração e do armazenamento. Este trabalho tem como objetivo avaliar o impacto da adoção de técnicas compensatórias, que promovem o aumento da capacidade de infiltração e retenção das águas pluviais, no controle dos escoamentos superficiais e de enchentes urbanas, com base em um estudo de caso de um pequeno loteamento residencial situado em Jacarepaguá, zona oeste da cidade do Rio de Janeiro. A metodologia envolveu a caracterização da área do loteamento, cálculo das vazões do escoamento superficial pelo método racional e dimensionamento das técnicas compensatórias pelo método da curva envelope para três cenários: (1) sem intervenção, (2) com implantação de trincheira de infiltração e, (3) com implantação de trincheira de infiltração e pavimento permeável. Os resultados indicaram vazões de 440, 200 e 30,2 L/s para os cenários (1), (2) e (3), respectivamente. 0 s valores obtidos demonstraram que somente a adoção das trincheiras de infiltração não seria totalmente efetivo na redução dos escoamentos superficiais, sendo a combinação das trincheiras de infiltração e adoção de pavimento permeável (cenário 3) a mais efetiva. 0s resultados evidenciam a importância da utilização das técnicas compensatórias de drenagem na gestão das águas pluviais, visto que reduzem significativamente o volume superficial escoado, sobretudo quando implantadas em conjunto.

Palavras-chave: Técnicas compensatórias, Enchentes urbanas, Controle do escoamento superficial
\end{abstract}

\section{The application of compensatory techniques on runoff control: case study of a residential allotment in Jacarepaguá, Rio de Janeiro}

\begin{abstract}
The city of Cuiabá may be considered privileged from the point of view of water resources. However, rapid urbanization and economic growth of the capital, from the 70s, affected the quality of these resources, especially its urban streams. In this context is inserted stream Engole Cobre, one of the 30 urban streams of Cuiabá. The aim of this study was to analyze the quality of stream Engole Cobra water and assess the level of environmental awareness of its surroundings residents. For the perception evaluation, a semi structured questionnaire was applied to record historical and social importance of the stream in these people lives. The physicochemical parameters evaluated for the water quality were $\mathrm{pH}$, conductivity, air and water temperatures and dissolved oxygen (D0). Residents remembered stream Engole Cobra of yore longingly. Its waters served to supply their homes, leisure and fishing, but now the stream is for them a concern due to its bad smell and the proliferation of vectors. Among the physicochemical parameters, the ones which best indicated the stream high degradation were, especially due to dumping of domestic waste, dissolved oxygen and conductivity, with average values of $1.99 \pm 0.42 \mathrm{mg} \mathrm{L}^{-1}$ and $482,83 \pm 111.32 \mu \mathrm{S} . \mathrm{cm}^{-1}$, respectively. It was concluded that stream Engole Cobra, like the other streams of the city, is neglected by the public management, requiring environmental education measures and collection and treatment of sewage from surrounding neighborhoods.
\end{abstract}

Keywords: Water quality, Urban streams, Basic sanitation, Domestic effluents, Dissolved oxygen. 


\title{
Aplicación de técnicas compensatorias en el control de las escorrentías superficiales: estudio de caso en una subdivisión residencial en Jacarepaguá, Río de Janeiro
}

\begin{abstract}
Resumen: El proceso de urbanización de las ciudades brasileñas dio como resultado una densificación de las zonas urbanas y, por consiguiente, el aumento de áreas impermeables, que favorecen la ocurrencia de inundaciones. Para compensar los efectos de la urbanización sobre el ciclo hidrológico se proponen medidas de control localizadas y de pequeña escala que promuevan una mayor infiltración y almacenamiento. Este estudio tiene como objetivo evaluar el impacto de la adopción de técnicas compensatorias en el control de la escorrentía y de las inundaciones urbanas, basado en un estudio de caso de una pequeña subdivisión residencial situada en Jacarepaguá, zona oeste de la ciudad de Río de Janeiro. La metodología consistió en la caracterización de la zona de adjudicación, el cálculo del flujo de escorrentía por el método racional y el diseño de técnicas compensatorias por el método de la curva envolvente para tres escenarios: (1) sin intervención, (2) con implementación de trinchera de infiltración y, (3) con implementación de trinchera de infiltración y pavimento permeable. Los resultados indicaron flujos de 400, 200 y 30,2 L/s para los escenarios (1), (2) y (3), respectivamente. Los valores muestran que sólo la adopción de trinchera de infiltración no sería totalmente eficaz en la reducción de la escorrentía, siendo la combinación de trinchera de infiltración y pavimento permeable la más eficaz. Los resultados evidencian la importancia de utilizar técnicas compensatorias de drenaje en la gestión de agua de lluvia, ya que reducen significativamente el volumen de escorrentía superficial, especialmente cuando implantadas en conjunto..

Palabras clave: Técnicas compensatorias, Inundaciones urbanas, Control de la escorrentía superficial
\end{abstract}

\section{INTRODUÇÃO}

0 rápido e expressivo aumento da população em centros urbanos, sobretudo nas últimas décadas do século XX, desencadeou mudanças no uso e na ocupação do solo, de modo que foram observadas alterações nas taxas de infiltração das águas pluviais no solo (ALMEIDA; FERREIRA NET0, 2015). A impermeabilização do solo devido às construções residenciais, telhados, estacionamentos, ruas pavimentadas e calçadas elevam as parcelas dos volumes de escoamento superficial, à medida que se reduz a capacidade de infiltração da água (OHNUMA JR e MENDI0ND0, 2015). Desta forma, a água que antes infiltrava no solo integra agora o escoamento superficial, sobrecarregando os sistemas de drenagem, e contribuindo para o aumento da frequência de inundações urbanas (OLIVEIRA, 2013).

Tendo em vista a consolidação no mundo inteiro do conceito de sustentabilidade a partir da década de 1970, a abordagem tradicional para sistemas de drenagem urbana passou por profunda revisão. Na atualidade, a água pluvial é reconhecida por seu valor como recurso natural que deve ser protegido e conservado. A nova concepção adota medidas que compensem as alterações do crescimento urbano no ciclo hidrológico, em direção à sustentabilidade (BAHIENSE, 2013). A drenagem urbana sustentável dispensa a ideia de rápido afastamento das águas pluviais e foca em condições que aumentem o tempo de concentração da bacia com uso de técnicas de armazenamento e infiltração. 
Aplicação de técnicas compensatórias no controle dos escoamentos superficiais: estudo de caso em loteamento residencial em Jacarepaguá, Rio de Janeiro

Este novo conjunto de técnicas integradoras foi uma evolução das chamadas "Best Management Practices" (BMPs) e traduz o conceito do Desenvolvimento de Baixo Impacto - Low Impact Development (LID), que foi evoluindo desde a década de 1980 e integram a gestão das águas pluviais nos Estados Unidos. As práticas LID são definidas como medidas de controle localizadas e de pequena escala, tendo como objetivo reproduzir as características naturais de uma região por meio de processos de infiltração, evaporação e filtração (DAMODARAM et al., 2010; LIU et al., 2015). Algumas destas práticas, também denominadas técnicas compensatórias, referem-se a dispositivos como os telhados verdes, pavimentos porosos, células de biorretenção, reservatórios de águas pluviais e trincheiras de infiltração.

Este trabalho tem como objetivo avaliar 0 impacto da adoção de técnicas compensatórias, que promovem o aumento da capacidade de infiltração e retenção das águas pluviais, no controle dos escoamentos superficiais e de enchentes urbanas, com base em um estudo de caso de um pequeno loteamento residencial situado na região da baixada de Jacarepaguá, zona oeste da cidade do Rio de Janeiro.

\section{MATERIAL E MÉTODOS}

\section{Caracterização da Área de Estudo}

A área de estudo refere-se a um loteamento residencial situado no bairro da Freguesia (Jacarepaguá), zona oeste da cidade do Rio de Janeiro, entre as coordenadas $22^{\circ} 56^{\prime} 5,86^{\prime \prime}$

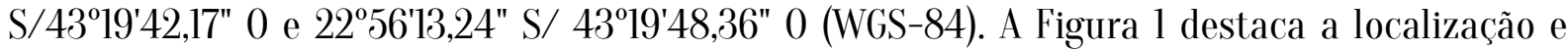
delimitação de tal loteamento, que encontra-se inserido na Área de Planejamento 4 (AP 4) do município do Rio de Janeiro. 0 mapa foi elaborado a partir do software ArcGIS 10.1 e da base de dados da Prefeitura da Cidade do Rio de Janeiro (IPP, 2015). 


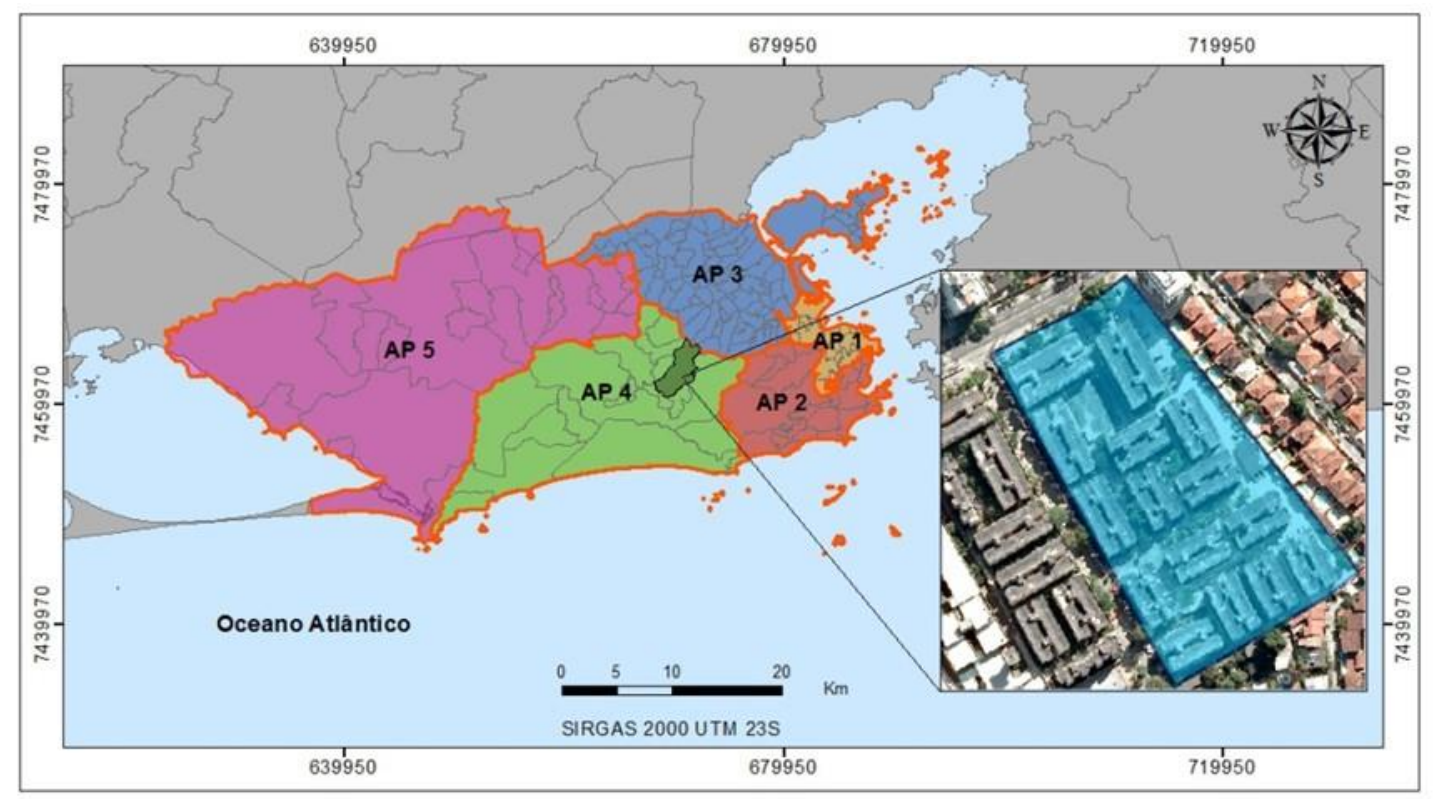

Figura 1. Localização da área de estudo situada no bairro da Freguesia (Jacarepaguá) Fonte: 0s autores, 2015.

A Freguesia apresenta uma taxa de ocupação do solo considerável, visto que ao longo dos últimos anos recebeu grandes investimentos imobiliários, além de um rápido desenvolvimento comercial. Este bairro compõe a baixada de Jacarepaguá, região de expansão e de grande importância para a cidade do Rio de Janeiro já que foi um dos locais de realização das Olimpíadas de 2016.

0 clima da baixada de Jacarepaguá caracteriza-se como equatorial de monções (tipo Am), segundo classificação de Köppen-Geiger, com verão chuvoso e inverno seco (RUBEL; KOTTEK, 2010).

A região da baixada de Jacarepaguá caracteriza-se também pela presença de wetlands naturais. Segundo Amaral (2014), no período chuvoso, principalmente no verão, as áreas alagadas ampliam e o transbordamento dos rios é recorrente. Com o acelerado desenvolvimento da região associado ao aumento da densidade populacional, expressivas alterações ocorreram na forma de ocupação do solo contribuindo para o agravamento das cheias naturais. 
Aplicação de técnicas compensatórias no controle dos escoamentos superficiais: estudo de caso em loteamento residencial em Jacarepaguá, Rio de Janeiro

\section{Técnicas Compensatórias Utilizadas no Estudo de Caso}

\section{Trincheiras de Infiltracão}

As trincheiras de infiltração são estruturas lineares que armazenam a água precipitada por tempo suficiente para sua infiltração no solo (PEITER; POLETO, 2012). Geralmente, conforme ilustrado na Figura 2, estas estruturas representam valas escavadas na terra com dimensões apropriadas, pouco profundas e preenchidas com material granular tais como brita e areia. Além disso, torna-se necessário a presença de manta geotêxtil entre o solo e material granular e, entre a superfície e a trincheira, de forma a evitar o processo de colmatação (deposição de partículas sólidas que comprometem o funcionamento do dispositivo) e a presença de elementos contaminantes.

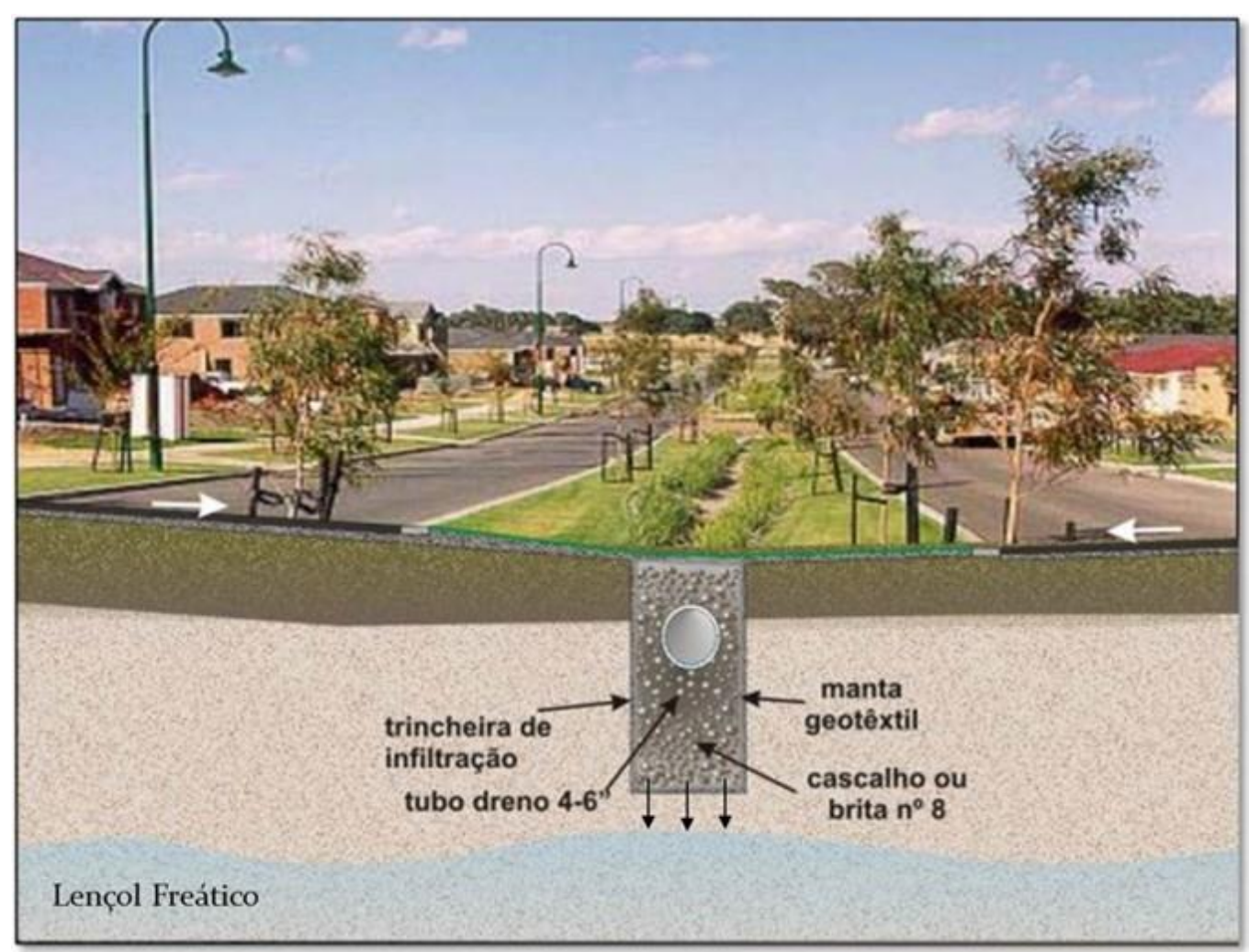

Figura 2. Trincheira de infiltração em canteiro central Fonte: Adaptado de GE0WATER, 2009.

As trincheiras de infiltração visam estabelecer, em parte, a infiltração das águas pluviais, componente do ciclo hidrológico que acaba comprometido devido à impermeabilização da superfície dos solos das áreas urbanas. Desta forma, sua principal vantagem está relacionada 
com a redução do volume escoado superficialmente, favorecendo a redução da ocorrência de inundações (GRACIOSA et al., 2008).

\section{Pavimento Poroso}

0 pavimento poroso, também chamado de pavimento permeável, representa outro tipo de dispositivo de controle na fonte e tem como objetivo a infiltração e/ou armazenamento da água, também promovendo a redução do volume escoado superficialmente (CASTR0 et al., 2013). Além disso, tais dispositivos auxiliam no controle da qualidade das águas pluviais já que 0 material que preenche o pavimento atua como filtro, de modo a reter as impurezas do volume infiltrado (BAHIENSE, 2013). A Figura 3 apresenta a estrutura típica para assentamento de pavimento constituído por concreto poroso. Logo abaixo da superfície dos blocos de concreto poroso observa-se uma camada de assentamento e rejunte constituída de agregado graúdo. Sendo que esta última se sobrepõe a uma camada constituída por reservatório de brita. Além do dimensionamento hidráulico, que mensura o volume de água a ser recebido e define os níveis de infiltração, os pavimentos são preparados para suportar certos tipos de carga mecânica (CORSINI, 2014).

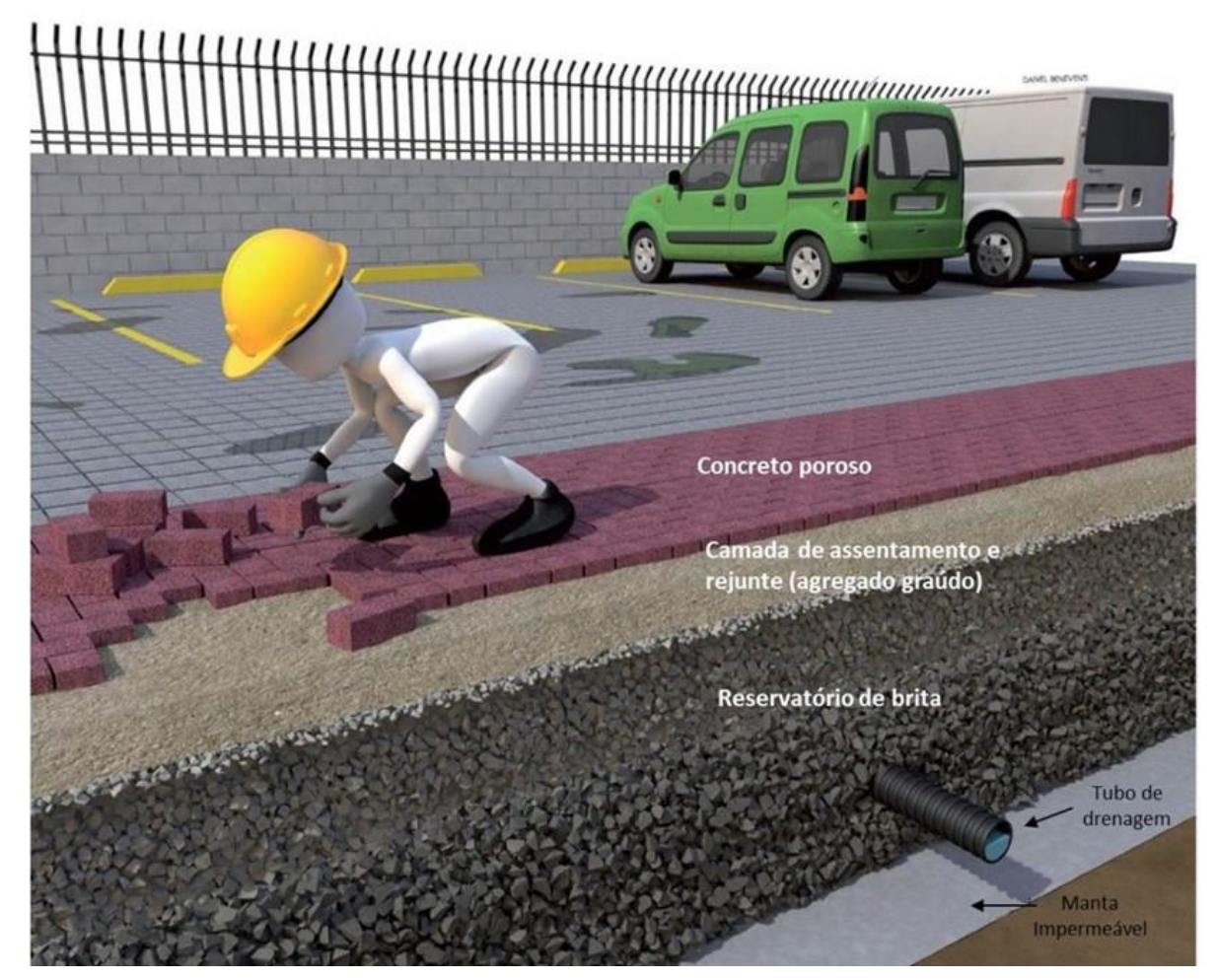

Figura 3. Pavimento permeável - sistema revestido com peças intertravadas de concreto poroso

Fonte: Adaptado de CORSINI, 2014. 
Aplicação de técnicas compensatórias no controle dos escoamentos superficiais: estudo de caso em loteamento residencial em Jacarepaguá, Rio de Janeiro

\section{Metodologia de Cálculo}

A quantificação e avaliação das vazões máximas de escoamento superficial geradas no loteamento consideraram os seguintes cenários: (1) situação atual da área de estudo; (2) implantação de trincheira de infiltração; (3) implantação de trincheira de infiltração e pavimento permeável. A metodologia para o cálculo das vazões foi baseada no Manual de Drenagem da Prefeitura da Cidade do Rio de Janeiro (RIO-ÁGUAS, 2010).

Com o auxílio do software AutoCAD 2015 e dos mapas digitais da Cidade do Rio de Janeiro (IPP, 2015), foi feita uma medição das áreas necessárias para o desenvolvimento do estudo de caso. Desta forma, o loteamento foi divido em 4 tipos de ocupação: pavimento (concreto), telhado, estacionamento e grama.

No cenário (1), a vazão máxima foi obtida pelo método racional, representado pela Equação l:

$$
Q=0.278 \mathrm{CiA}
$$

Onde:

Q = vazão máxima (de pico do hidrograma), $\mathrm{em} \mathrm{m}^{3} / \mathrm{s}$;

$\mathrm{C}$ = coeficiente de runoff ou de escoamento superficial, adimensional;

i = intensidade pluviométrica, em $\mathrm{mm} / \mathrm{h}$;

$\mathrm{A}=$ área, $\mathrm{em} \mathrm{km²}$.

Para os valores dos coeficientes de runoff $(\mathrm{C})$ foram adotados os valores recomendados em ASCE (1969).

A intensidade pluviométrica foi obtida a partir da equação de chuvas intensas (Equação 2), conforme Rio-Águas (2010).

$$
i=\frac{a \times T R^{b}}{(t+c)^{d}}
$$


Onde:

i = intensidade pluviométrica, em $\mathrm{mm} / \mathrm{h}$;

TR = tempo de recorrência, em anos;

$\mathrm{t}$ = duração da chuva, em minutos;

a, b, c, d: valores dos coeficientes atribuídos conforme a localização da área em estudo.

0 tempo de recorrência (TR) e duração da chuva de projeto $(\mathrm{t})$ correspondem a 2 anos e 15 minutos, respectivamente, conforme sugestão da Rio-Águas (2010) para projetos de micro drenagem.

0 dimensionamento da trincheira de infiltração e do pavimento permeável foi realizado com base no método da curva envelope (DAEE/CETESB, 1980), geralmente aplicado no dimensionamento de bacias de detenção. Este método consiste em comparar os volumes afluente (de entrada) e efluente (de saída) no tempo, sendo a diferença correspondente ao volume de armazenamento do dispositivo.

A Tabela 1 apresenta as principais equações de pré-dimensionamento das estruturas de controle utilizadas neste estudo.

Tabela 1 - Equações de pré-dimensionamento da trincheira de infiltração e pavimento poroso

\begin{tabular}{|c|c|c|}
\hline Dispositivo & Equações & Variáveis \\
\hline \multirow{6}{*}{$\begin{array}{l}\text { Trincheira de } \\
\text { Infiltração }\end{array}$} & $V t r=V a r m z / \varphi_{b}(3)$ & $\mathrm{V}_{\text {tr: }}$ volume da trincheira $\left(\mathrm{m}^{3}\right)$ \\
\hline & & $\mathrm{V}_{\text {armz: }}$ volume armazenado $\left(\mathrm{m}^{3}\right)$ \\
\hline & Varmz $=$ Vprec $-\operatorname{Vinf}(4)$ & 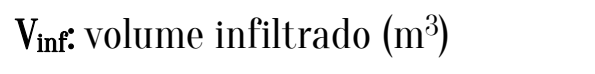 \\
\hline & & $\begin{array}{l}\boldsymbol{\varphi}_{\mathrm{b}}: \text { porosidade do material } \\
\text { granular }\end{array}$ \\
\hline & Vprec $=Q \times \Delta t(5)$ & $\begin{array}{l}\text { Q: vazão afluente à trincheira } \\
\left(\mathrm{m}^{3} / \mathrm{s}\right)\end{array}$ \\
\hline & $\operatorname{Vinf}=H \times L \times I a c(6)$ & $\Delta \mathrm{t}$ : duração da precipitação (s) \\
\hline
\end{tabular}




\begin{tabular}{|c|c|c|}
\hline & \multirow{4}{*}{$I a c=S \sqrt{ } \Delta t(7)$} & H: altura da trincheira (m) \\
\hline & & L: comprimento da trincheira $(\mathrm{m})$ \\
\hline & & $\mathrm{I}_{\mathrm{ac}}$ : infiltração acumulada $\left(\mathrm{m}^{3} / \mathrm{m}^{2}\right)$ \\
\hline & & S: sortividade do solo $\left(\mathrm{m}^{3} / \mathrm{s} . \mathrm{m}^{2}\right)$ \\
\hline \multirow{11}{*}{$\begin{array}{l}\text { Pavimento } \\
\text { Permeável }\end{array}$} & \multirow{3}{*}{$\begin{aligned} V \max =\left(\sqrt{\frac{a}{60}}\right. & \times \sqrt{\beta} \times T^{b / 2} \\
& \left.-\sqrt{\frac{c}{60}} \times \sqrt{q_{s}}\right)^{2}\end{aligned}$} & $\mathrm{~V}_{\text {max: }}$ volume armazenado $(\mathrm{mm})$ \\
\hline & & $\begin{array}{l}\text { a, b, c: parâmetros da equação IDF } \\
\text { de Talbot }\end{array}$ \\
\hline & & T: período de retorno (anos) \\
\hline & \multirow[t]{2}{*}{$q s=\propto \times K s(9)$} & $\begin{array}{l}\text { qs: taxa de infiltração do solo } \\
(\mathrm{mm} / \mathrm{h})\end{array}$ \\
\hline & & $\begin{array}{l}\boldsymbol{\alpha}: \text { coeficiente redutor devido à } \\
\text { colmatação }\end{array}$ \\
\hline & \multirow{2}{*}{$\beta=\frac{A_{p a v}-A \times C_{e}}{A_{p a v}}$} & $\begin{array}{l}\mathbf{k}_{\mathrm{s}:} \text { condutividade hidráulica do solo } \\
(\mathrm{mm} / \mathrm{h})\end{array}$ \\
\hline & & 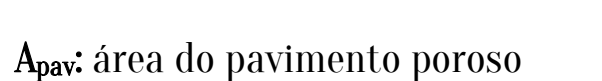 \\
\hline & (10) & $\begin{array}{l}\text { A: área que contribui para } 0 \\
\text { pavimento poroso }\end{array}$ \\
\hline & \multirow{2}{*}{$H=\frac{V_{\max }}{\eta}$} & $\mathrm{C}_{\mathrm{e}}$ : coeficiente de runoff \\
\hline & & H: altura do reservatório $(\mathrm{mm})$ \\
\hline & (11) & 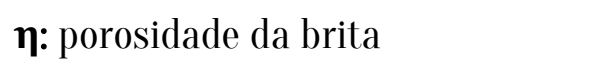 \\
\hline
\end{tabular}

Fonte: SILVEIRA e GOLDENFUM, 2007.

Apesar do decreto $\mathrm{n}^{\circ} 23.940$ de 30 de janeiro de 2004 obrigar 0 armazenamento temporário de águas pluviais em lotes com mais de $500 \mathrm{~m}^{2}$ de área construída, a Cidade do Rio de Janeiro não conta com legislação específica para volumes máximos admitidos de escoamento superficial pós-urbanização. Desta forma, foi considerado como referência o decreto $\mathrm{n}^{0} 15.371$ de 17 de novembro de 2006, que estabelece o valor de 20,8 L/(s.ha) como vazão máxima específica de saída para o sistema de drenagem da Cidade de Porto Alegre. 
Para aplicação do método da curva envelope, em especial a Equação 8, é preciso fazer a conversão dos parâmetros da curva IDF convencional para a expressão de Talbot. As Equações 14, 15 e 16, apresentadas na Tabela 2, foram aplicadas na conversão dos valores dos parâmetros.

Tabela 2 - Expressões de conversão dos parâmetros da equação IDF

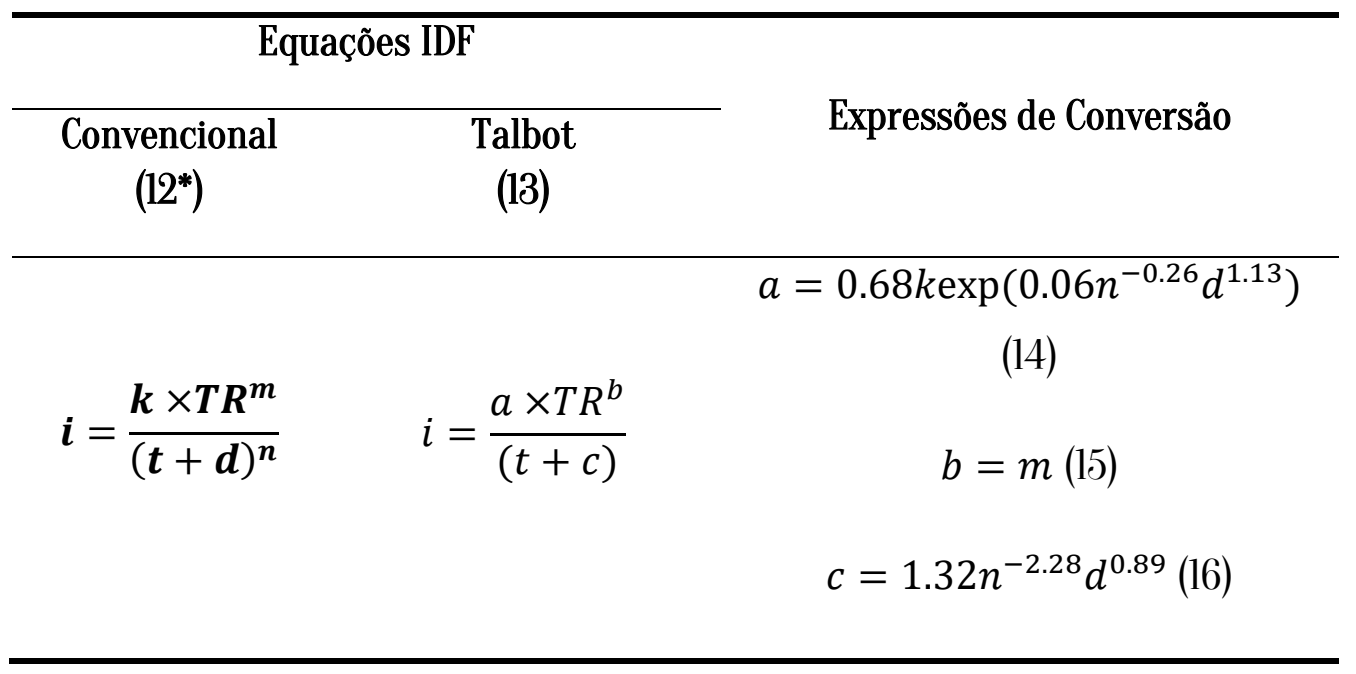

* Equivalente à Equação 2.

Fonte: : SILVEIRA e GOLDENFUM, 2007.

Segundo o mapa de solos do Rio de Janeiro, escala de 1:5.000.000, disponibilizado pelo IBGE (2001), o solo da região de estudo é do tipo Argissolo Vermelho-Amarelo. Esta informação é importante para a obtenção da condutividade hidráulica e sortividade do solo, parâmetros necessários para dimensionamento da trincheira de infiltração e pavimento permeável 
Aplicação de técnicas compensatórias no controle dos escoamentos superficiais: estudo de caso em loteamento residencial em Jacarepaguá, Rio de Janeiro

\section{RESULTADOS E DISCUSSÃ0}

Os valores encontrados para cada ocupação do loteamento são apresentados na Tabela 3.

Tabela 3 - Áreas de parcelamento do loteamento por tipologia de uso e ocupação do solo

\begin{tabular}{ccc}
\hline \multirow{2}{*}{ Tipo } & \multicolumn{2}{c}{ Área } \\
\cline { 2 - 3 } & $\mathrm{m}^{2}$ & \% \\
\hline Concreto & 7197,3 & 39,68 \\
Estacionamento & 2089,0 & 11,52 \\
Telhado & 7037,0 & 38,80 \\
Grama & 1813,7 & 10,00 \\
TOTAL & 18137,0 & 100,00 \\
\hline
\end{tabular}

Fonte: 0s autores, 2015.

Para cada área do loteamento foi adotado um coeficiente de runoff (C), conforme apresentado na Tabela 4.

Tabela 4 - Valores do coeficiente runoff $(\mathrm{C})$

\begin{tabular}{cccc}
\hline & Superfície & Intervalo & Valor adotado \\
\hline Pavimento & Concreto & $0.80-0.95$ & 0.90 \\
& Telhado & $0.75-0.95$ & 0.85 \\
Cobertura & $\begin{array}{c}\text { Grama solo arenoso } \\
\text { (pequena } \\
\text { declividade) }\end{array}$ & $0.05-0.10$ & 0.10 \\
\hline
\end{tabular}

Fonte: ASCE, 1969.

Os coeficientes necessários para a aplicação da Equação 2, em função da localização do pluviômetro mais próximo do loteamento, estão apresentados na Tabela 5. 
Tabela 5 - Coeficientes para a Curva IDF da região do estudo de caso

\begin{tabular}{ccccc}
\hline Pluviômetro & A & B & C & D \\
\hline Viall (Jacarepaguá) & 1.423 & 0,196 & 14,58 & 0,796 \\
\hline
\end{tabular}

Fonte: RI0-ÁGUAS, 2010.

\section{Cenário (1): sem intervenção}

Utilizando a equação IDF, com os parâmetros indicados na Tabela 5, obteve-se uma intensidade pluviométrica de $109,75 \mathrm{~mm} / \mathrm{h}$, correspondente a uma chuva com duração de 15 minutos e período de retorno de 2 anos.

0 valor de C (coeficiente de escoamento) para o loteamento foi obtido pela média ponderada dos valores de C (ASCE, 1969) para cada área de parcelamento do loteamento segundo tipologia de uso e ocupação do solo (Tabela 3). 0 peso de cada parcela no cálculo da média foi calculado pela razão entre a área da parcela e a área total do loteamento. 0 valor final obtido para C, representativo de toda área, foi de 0,80. Aplicando-se o método racional (Equação 1), a vazão máxima (de pico) gerada pelo loteamento é de $0,44 \mathrm{~m}^{3} / \mathrm{s}$ ou $440 \mathrm{~L} / \mathrm{s}$.

0 cenário 1 revela que a vazão de escoamento superficial é superior ao valor de referência utilizado pelo presente trabalho, com base no decreto $\mathrm{n}^{0}$ 15.371 (PORTO ALEGRE, 2006). Para atender ao valor de $20,8 \mathrm{~L} /$ (s.ha) ou $37,65 \mathrm{~L} / \mathrm{s}$ para a área total do loteamento, foram aplicadas as técnicas compensatórias de trincheira de infiltração e pavimento poroso (permeável), conforme apresentado a seguir.

\section{Cenário (2): implantação de trincheira de infiltração}

Na Figura 4 é apresentado o local escolhido para implantação da trincheira de infiltração. 


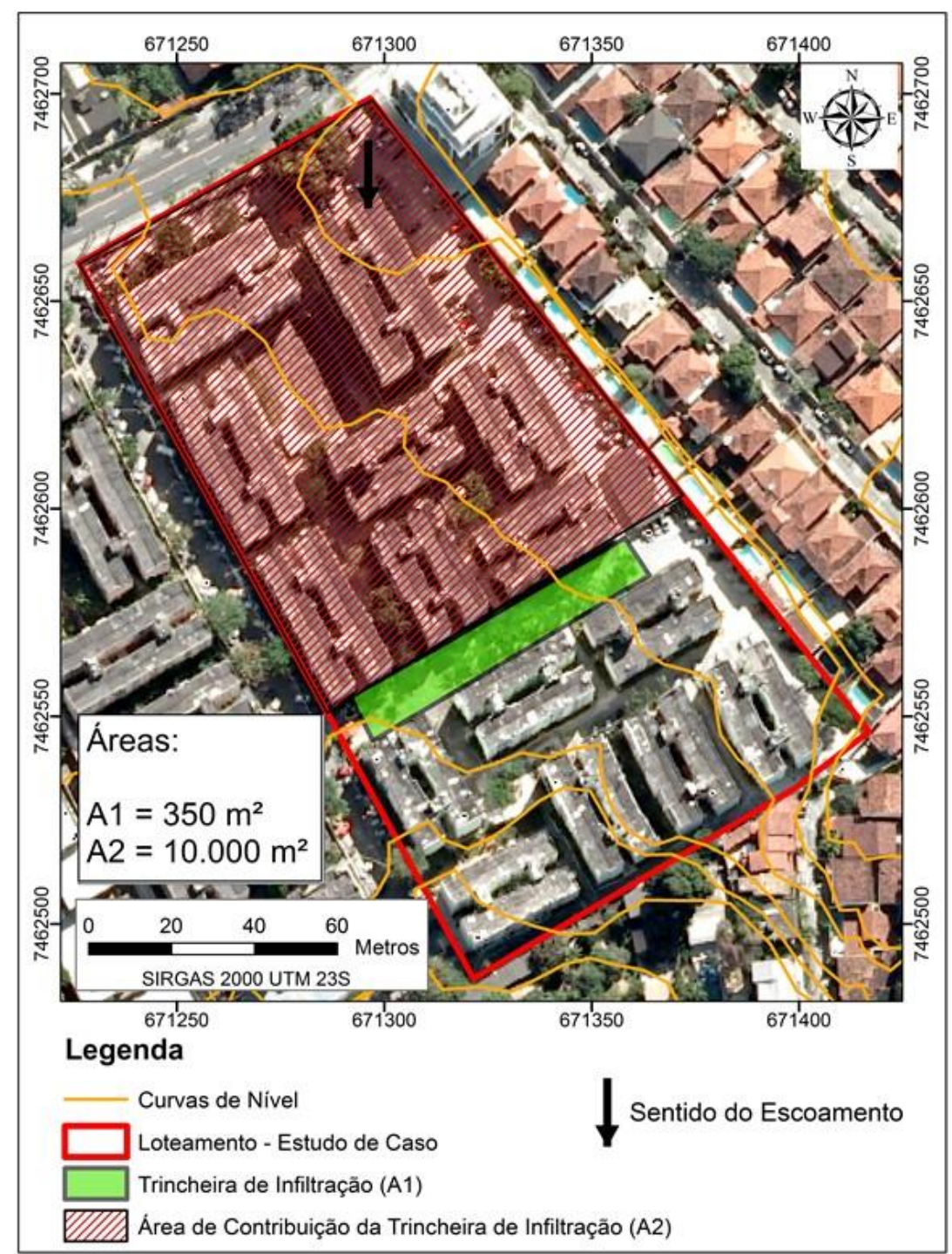

Figura 4. Localização da trincheira de infiltração Fonte: 0s autores, 2015.

Em decorrência do sentido do escoamento superficial, a área que contribui para a trincheira de infiltração é de aproximadamente $10.000 \mathrm{~m}^{2}$ ou 1 ha.

0ptou-se por dimensionar uma trincheira com $5 \mathrm{~m}$ de largura e $70 \mathrm{~m}$ de comprimento, para armazenar a vazão de escoamento superficial gerada pelos $10.000 \mathrm{~m}^{2}$ da área de contribuição. Essa vazão foi obtida pelo método racional, utilizando o coeficiente de runoff ponderado de 0,80 , resultando em $0,24 \mathrm{~m}^{3} / \mathrm{s}$ ou $240 \mathrm{~L} / \mathrm{s}$.

Na ausência de um estudo das propriedades físicas do solo do loteamento, utilizou-se os dados experimentais obtidos por Graciosa et al. (2008) para dimensionamento de uma trincheira 
de infiltração em dois tipos de solos da cidade de São Carlos - SP. A partir deste estudo, adotouse 0 valor de sortividade igual a $0,00571 \mathrm{~m}^{3} / \mathrm{m}^{2}$. s.

Aplicando-se as equações descritas na Tabela 1, para o dimensionamento da trincheira de infiltração, foram obtidos os seguintes valores:

$$
\begin{aligned}
& \mathrm{I}_{\mathrm{acm}}=0,17 \mathrm{~m}^{3} / \mathrm{m}^{2} ; \\
& V_{\mathrm{inf}}=15,59 \mathrm{~m}^{3} ; \\
& V_{\mathrm{armz}}=204,26 \mathrm{~m}^{3} .
\end{aligned}
$$

Optou-se por um leito de brita com porosidade de $45 \%$, resultando em um $\mathrm{V}_{\mathrm{tr}}=453,91 \mathrm{~m}^{3}$. Para atender a esse volume de trincheira, foi necessário utilizar uma altura de 1,3 m. Portanto, o dimensionamento da trincheira para armazenar 0 volume referente à vazão de $0,24 \mathrm{~m}^{3} / \mathrm{s}$ durante 15 minutos é:

$$
\begin{aligned}
& \mathrm{L}=70 \mathrm{~m} \text { (comprimento); } \\
& \mathrm{B}=5,0 \mathrm{~m} \text { (largura); } \\
& \mathrm{H}=1,3 \mathrm{~m} \text { (altura). }
\end{aligned}
$$

Nesse caso, o loteamento irá gerar escoamento superficial apenas na área ao sul da trincheira de infiltração. Essa vazão, obtida pela diferença da vazão gerada pelo loteamento e da vazão amortecida pela trincheira, corresponde a $200 \mathrm{~L} / \mathrm{s}$.

Desta forma, a implantação da trincheira de infiltração no local admitido não é suficiente para atender os critérios do Decreto de Porto Alegre, e foi admitida a adição de outra técnica compensatória para o amortecimento da vazão escoada superficialmente. 
Aplicação de técnicas compensatórias no controle dos escoamentos superficiais: estudo de caso em loteamento residencial em Jacarepaguá, Rio de Janeiro

\section{Cenário (3): implantação de trincheira de infiltração e pavimento permeável}

Neste cenário, além da utilização da trincheira de infiltração como técnica compensatória, idealiza-se uma área de pavimento permeável a fim de diminuir ainda mais a vazão a ser lançada nas redes pluviais.

0 esquema da Figura 5 apresenta as áreas a serem utilizadas por cada técnica.

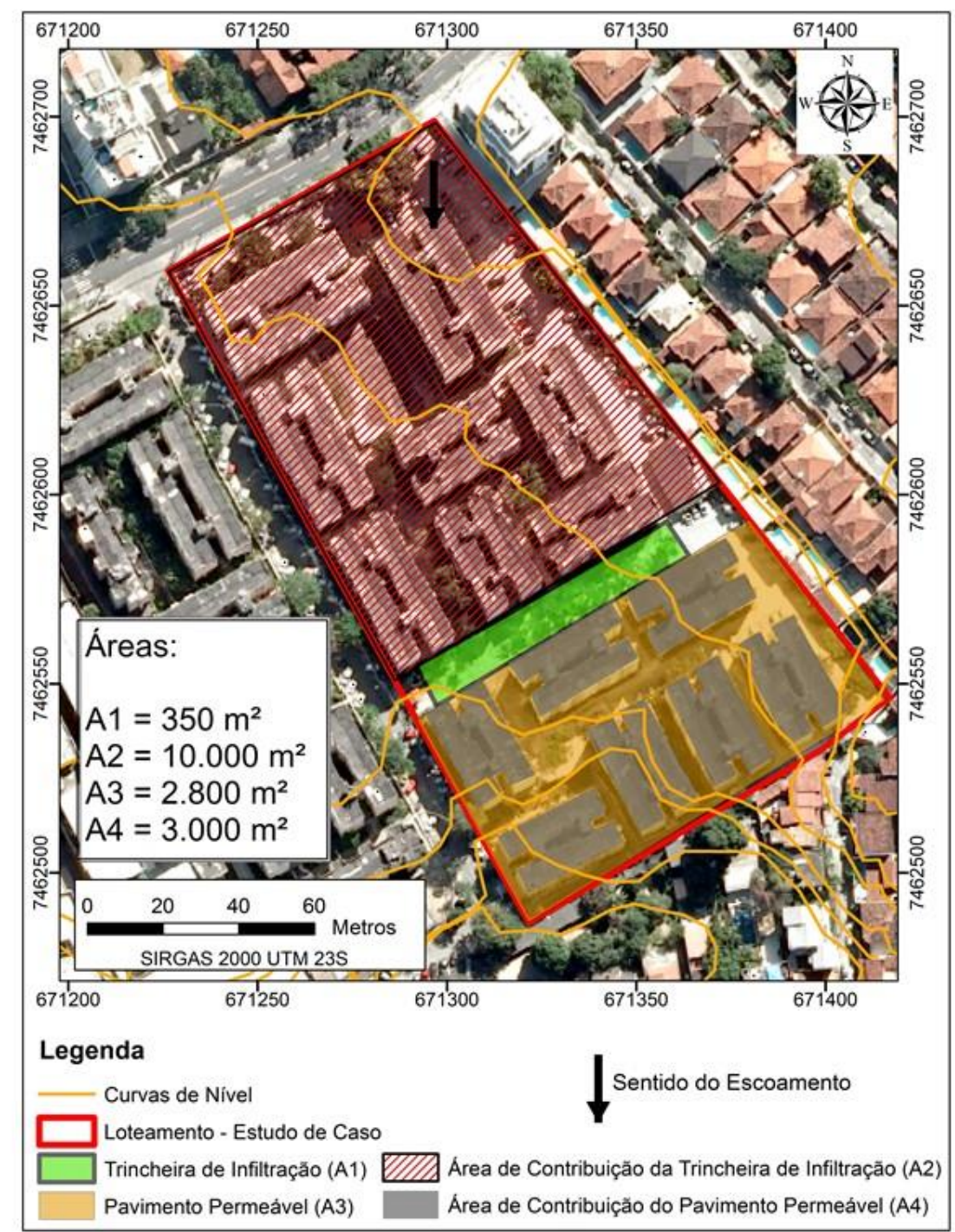

Figura 5. Localização da trincheira de infiltração e pavimento permeável Fonte: Os autores, 2015.

De acordo com Santos e Castro (2006), para Argissolo Vermelho-Amarelo obteve-se um valor de condutividade hidráulica igual a $6 \mathrm{~mm} / \mathrm{h}$. Além disso, adotou-se um coeficiente de segurança de redução da taxa de infiltração de 0,1, segundo CIRIA (1996), por ser uma área que 
pode ocasionar muitos prejuízos devido às enchentes, visto que é uma região densamente povoada e de baixada, como especificado na introdução do presente trabalho.

A área de contribuição do pavimento permeável corresponde à área dos telhados que não drenaram as águas para a trincheira, sendo este valor de aproximadamente $3.000 \mathrm{~m}^{2}$. A área de pavimento utilizada para o dimensionamento é igual a $2.800 \mathrm{~m}^{2}$.

Como já dito, a aplicação do método da curva envelope para dimensionamento de pavimentos porosos requer a transformação dos coeficientes da equação IDF convencional para a equação de Talbot. Esta conversão foi feita com base nas expressões da Tabela 2 e os coeficientes obtidos estão listados na Tabela 6.

Tabela 6 - Parâmetros da equação IDF de Talbot

\begin{tabular}{cc}
\hline & IDF Talbot \\
\hline a & 3575,33 \\
b & 0,19 \\
c & 23,99 \\
\hline
\end{tabular}

Fonte: 0s Autores, 2015.

Ao aplicar as fórmulas da Tabela 1, obtiveram-se os seguintes valores para os parâmetros necessários ao dimensionamento:

$\mathrm{q}_{\mathrm{s}}=0,6 \mathrm{~mm} / \mathrm{h}$;

$\beta=1,964$

$\mathrm{V}_{\max }=122,44 \mathrm{~mm}$.

Ao utilizar uma brita que tenha 45\% de porosidade, encontrou-se o valor de $28 \mathrm{~cm}$ para a altura do reservatório. 
Aplicação de técnicas compensatórias no controle dos escoamentos superficiais: estudo de caso em loteamento residencial em Jacarepaguá, Rio de Janeiro

A geração de escoamento superficial se dará apenas pela área composta por grama, localizada ao sul da trincheira, que corresponde a 5\% da área total do loteamento, ou seja, 906,85 $\mathrm{m}^{2}$, além de uma área do estacionamento equivalente a 1080,15 m².

0 método racional foi aplicado para as duas áreas, sendo o coeficiente de runoffigual a 0,10 para a área verde e 0,90 para o estacionamento, e obteve-se as vazões de 2,75 L/s e 27,46 $\mathrm{L} / \mathrm{s}$, respectivamente. A soma das duas vazões equivale a $30,21 \mathrm{~L} / \mathrm{s}$.

Portanto, um pavimento com $2.800 \mathrm{~m}^{2}$ de área, com uma camada porosa de $28 \mathrm{~cm}$ de brita com porosidade de 45\%, além da trincheira de infiltração anteriormente dimensionada, leva a um escoamento superficial de $30,21 \mathrm{~L} / \mathrm{s}$, atendendo aos $37,65 \mathrm{~L} / \mathrm{s}$ propostos no presente trabalho.

A Tabela 7 apresenta de forma consolidada os valores de vazões geradas em cada cenário.

Tabela 7 - Parâmetros da equação IDF de Talbot)

\begin{tabular}{cc}
\hline Cenários & Vazão Gerada (L/s) \\
\hline (1) Sem intervenção & 440,0 \\
(2) Trincheira de Infiltração & 200,0 \\
(3) Trincheira de Infiltração e Pavimento & 30,2 \\
Permeável & \\
\hline
\end{tabular}

Fonte: 0s Autores, 2015

\section{CONCLUSÃO}

As técnicas compensatórias mostram-se boas soluções para o amortecimento do escoamento superficial em loteamentos que, devido à impermeabilização do solo, aumentou substancialmente em relação às condições anteriores à urbanização. Tais técnicas ainda auxiliam no melhoramento da qualidade da água entregue aos corpos hídricos receptores.

Quanto aos resultados apresentados, verificou-se uma redução de 55\% da vazão escoada em relação ao cenário (1), considerando a implantação de uma trincheira de infiltração para 0 amortecimento do escoamento superficial. Já no cenário (3), constatou-se uma redução de 93\% da vazão em comparação com a vazão gerada pelo loteamento sem intervenções. 
Tais reduções exprimem a importância de se utilizar técnicas compensatórias como auxílio ao manejo sustentável das águas pluviais. E, ainda, mostram que as técnicas podem trabalhar em conjunto para uma diminuição do escoamento superficial, principalmente quando há condicionantes que impeçam a utilização ampla de apenas uma técnica, seja por inviabilidade econômica ou técnica.

Sugere-se que a cidade do Rio de Janeiro discuta e elabore normas para limitar o escoamento máximo dentro de loteamentos, no intuito de amenizar os impactos decorrentes de eventos pluviométricos extremos, que são amplificados pela ocupação de solo sem planejamento nas áreas urbanas.

Para trabalhos futuros, recomenda-se o estudo da implantação de telhados verdes e reservatórios como alternativas de armazenamento de água pluvial, visto que a área de coberturas e telhados no meio urbano é significativa e conta com um coeficiente de runoff elevado.

\section{REFERÊNCIAS BIBLIOGRÁFICAS}

ALMEIDA, A. J. P.; FERREIRA NETO, J. V. Análise do processo de urbanização e impermeabilização do solo da bacia endorreica do tabuleiro do Martins, Maceió-AL. In: Anais do XI Encontro Nacional da ANPEGE, São Paulo, 2015.

AMARAL, D. P. B. Aplicação do modelo hidrológico SWMM na gestão das águas pluviais urbanas: estudo de caso da cacia hidrográfica do Rio Morto. 2014. Dissertação (Mestrado) - Faculdade de Engenharia, Universidade do Estado do Rio de Janerio, Rio de Janeiro.

ASCE. Design and Construction of sanitary and storm sewers. New York (Manuals and Reports of Engineering Practice, 37), 1969.

BAHIENSE, J. M. Avaliação de Técnicas Compensatórias em Drenagem Urbana Baseadas no Conceito de Desenvolvimento de Baixo Impacto, com o Apoio de Modelagem Matemática. 2013. Dissertação (Mestrado em Engenharia Civil) - Universidade Federal do Rio de Janeiro - COPPE, Rio de Janeiro.

CASTRO, A. S. et al. Avaliação da Evolução do Comportamento Quantitativo de Pavimentos Permeáveis no Controle do Escoamento Superficial. Revista Brasileira de Recursos Hídricos, v.18, n.l, p. 263-273, 2013.

CIRIA. Infiltration drainage - Manual of good practice. Roger Bettes Bsc PhD MCIWEM. CIRIA Report 156. 1996.

CORSINI, R. Pavimento Permeável - Sistema revestido compeças interravadas de concreto poroso promovem a infiltração de água. 2014. Disponível em: http://infraestruturaurbana.pini.com.br/solucoestecnicas/37/pavimento-permeavel-308773-1.aspx. Acessado em 15 nov 2015.

DAEE/CETESB. Drenagem Urbana, Manual de Projeto, Departamento de Águas e Energia Elétrica e Companhia de Tecnologia de Saneamento, São Paulo, 1980.

DAMODARAM, C. et al. Simulation of Combined Best Management Practices and Low Impact Development for Sustainable Stormwater Management. Journal of the American Water Resources Association, v.46, n.5, p. 907-918, 2010. 
GEOWATER. Anexo 2: Ilustrações de Sistemas de Manejo Sustentável de Águas Pluviais. Plano Municipal de Saneamento de Macatuba, 2009. Disponível em: https://camaramm.files.wordpress.com/2009/10/ilustracoes-desistemas-de-manejo-sustentavel-de-aguas-pluviais.pdf. Acessado em 15 nov 2015.

GRACIOSA, M. C. P. et al. Metodologia para o Dimensionamento de Trincheiras de Infiltração para o Controle do Escoamento Superficial na Origem. Revista Brasileira de Recursos Hídricos, v.13, n.2, p. 207-214, 2008.

IBGE. EMBRAPA - Mapa de Solos do Brasil. Rio de Janeiro: IBGE, 2001 - Escala 1:5.000.000. Disponível em: $\langle$ http://www.dpi.inpe.br/Ambdata/mapa_solos.php〉.

IPP. Instituto Pereira Passos. Armazém de Dados. Mapa Digital do Rio de Janeiro. Base Geoweb - Extração de Base de Dados $\quad$ Geográficos. $\quad$ Disponível em: http://portalgeo.rio.rj.gov.br/mapa_digital_rio/?config=config/ipp/basegeoweb.xml. Acessado em 14 Jul 2015.

LIU, Y. et al. Enhancing a rainfall-runoff model to assess the impacts of BMPs and LID practices on storm runoff. Journal of Environmental Management, v.147, p. 12-23, 2015.

OLIVEIRA, D. F. R. 0 risco de inundação urbana nas frentes de água de deltas e estuários em cenários de alterações climáticas. 2013. Dissertação (Mestrado) - Instituto Superior de Agronomia, Universidade Técnica de Lisboa, Lisboa.

OHNUMA JR, A. A.; MENDIOND0, E. M. Metodologia para cálculo de eficiência de técnicas compensatórias em lote urbano. Revista Internacional de Ciências, v.5, n.1, p. 29-41, 2015.

PEITER, T. V.; POLETO, C. Estudos dos efeitos de trincheiras de infiltração sobre o escoamento superficial. Revista de Estudos Ambientais, v.14, n.2, p. 57-67, 2012.

PORTO ALEGRE. Decreto $\mathrm{n}^{0} 15.371$ de 17 de novembro de 2006. Regulamenta o controle da drenagem urbana. Porto Alegre, 2006.

RI0-ÁGUAS. Instruções Técnicas para Elaboração de Estudos Hidrológicos e Dimensionamento Hidráulico de Sistemas de Drenagem Urbana. Subsecretaria de Gestão de Bacias Hidrográficas - Rio-Águas, $1^{\mathrm{a}}$ versão, 2010.

RUBEL, F.; KOTTEK, M. Observed and projected climate shifts 1901-2100 depicted by world maps of the KöppenGeiger climate classification. Meteorologische Zeitschrift, v. 19, n.2, p. 135-141, 2010.

SANTOS, L. J. C.; CASTRO, S. S. Lamelas (bandas onduladas) em argissolo vermelho-amarelo como indicadores da evolução do relevo: o caso das colinas médias do platô de Bauru (SP). Revista Brasileira de Geomorfologia, Ano 7, n.l, p. 43-64, 2006.

SILVEIRA, A. L. L.; GOLDENFUM, J. A. Metodologia generalizada para pré-dimensionamento de dispositivos de controle pluvial na fonte. Revista Brasileira de Recursos Hídricos, v. 12, n. 2, p. 157-168, 2007. 